\title{
Alpha thalassaemia and the macular vasculature in homozygous sickle cell disease
}

\author{
P. I. CONDON, R. J. MARSH, G. H. MAUDE, D. R. HIGGS, \\ D. J. WEATHERALL, AND G. R. SERJEANT
}

From the Medical Research Council Laboratories, University of the West Indies, Jamaica

SUMMARY The interaction of homozygous alpha thalassaemia 2 with homozygous sickle cell (SS) disease results in a generally milder haematological picture with less intravascular sickling, less haemolysis, and higher haemoglobin levels. Clinically, patients are generally more mildly affected, though not all vaso-occlusive complications are reduced. Thus there is a possibility that the advantages gained by inhibition of sickling have been offset by the rheological disadvantages of the higher haemoglobin level. The capillary bed in the perimacular region of the posterior pole has been used to examine the degree of vaso-occlusion in age and sex matched controls with SS disease with and without homozygous alpha thalassaemia 2 . The results demonstrated significantly less capillary abnormalities in the perimacular region of patients with alpha thalassaemia, though the size of the foveal avascular zone and the grading of perimacular capillary drop-out did not differ between the 2 genotypes. These results are compatible with a mild inhibitory effect of alpha thalassaemia on vaso-occlusion of the macular vasculature in SS disease.

The clinical and haematological features of homozygous sickle cell (SS) disease vary markedly between patients. Part of this variability is now known to result from the interaction of SS disease with alpha thalassaemia $2 .{ }^{12}$ Patients with SS disease who are also homozygous for alpha thalassaemia 2 (SS $\alpha$-/ $\alpha$-) have milder haematological abnormalities with higher total haemoglobin levels, less evidence of haemolysis, and lower irreversibly sickled cell (ISC) counts compared with matched controls with a normal number of alpha globin genes (SS $\alpha \alpha / \alpha \alpha)$. There was also some clinical amelioration with significantly less acute chest syndrome and less chronic leg ulceration. However, other complications with a presumed vaso-occlusive component (painful crises, priapism, fetal loss) were not significantly different between SS patients with and without alpha thalassaemia $2 .{ }^{2}$

In view of the conflicting evidence on vasoocclusion in SS $\alpha$ - $/ \alpha$-, it has been postulated that some of the rheological advantages of inhibition of in-vivo sickling are offset by the increased viscosity associated with higher haemoglobin levels. This hypothesis has

Correspondence to Dr G. R. Serjeant, Medical Research Council Laboratories, University of the West Indies, Mona, Kingston 7, Jamaica, W.I. been tested by direct observation of the degree of vaso-occlusion in the posterior polar capillary bed in age and sex matched pairs of SS patients with and without alpha thalassaemia 2 .

\section{Materials and methods}

The patients attended the sickle cell clinic of the University Hospital of the West Indies, Kingston, Jamaica and a group of peripheral sickle cell clinics operated by the staff of the MRC Laboratories. The diagnosis of SS disease and of the alpha thalassaemia genotype was made as previously described. ${ }^{2}$ From the original study of 44 patients homozygous for alpha thalassaemia 2 and 88 matched controls with a normal alpha globin gene complement, appointments for ophthalmological examination were given to 25 pairs (patient and first control) during a one-week period in February 1982. Of these, 19 pairs attended and adequate posterior polar angiograms were obtained in 14 pairs (10 female; 4 male). Ages ranged from 10 to 44 years, median $21 \cdot 5$ years.

Ophthalmological examination was performed as previously described ${ }^{3}$ with angiography of the macular area of both eyes. Vascular abnormalities occurred at the posterior pole in all patients, and 3 
Table 1 Size of foveal avascular zone (FAZ), grading of perimacular avascular zones (PAZ), and counts of perimacular capillary abnormalities (perimacular counts) in patients with SS disease with (SS $\alpha$-/ $\alpha$-) and without (SS $\alpha \alpha / \alpha \alpha)$ interacting alpha thalassaemia

\begin{tabular}{|c|c|c|c|c|c|}
\hline \multirow[t]{2}{*}{ Index } & \multicolumn{2}{|l|}{ Mean (range) } & \multirow{2}{*}{$\begin{array}{l}\text { Paired } t \\
\text { statistic }\end{array}$} & \multirow[t]{2}{*}{$D F$} & \multirow[t]{2}{*}{ Significance } \\
\hline & $S S \alpha-/ \alpha-$ & $S S \alpha \alpha / \alpha \alpha$ & & & \\
\hline FAZ diameter (mm) & $\begin{array}{c}0.63 \\
(0 \cdot 50-0 \cdot 80)\end{array}$ & $\begin{array}{c}0.62 \\
(0.46-0.69)\end{array}$ & -0.53 & 12 & NS \\
\hline PAZ score & $\begin{array}{r}1 \cdot 6 \\
(1-3)\end{array}$ & $\begin{array}{l}1 \cdot 4 \\
(1-2)\end{array}$ & 0.63 & 11 & NS \\
\hline Perimacular counts & $\begin{array}{l}157 \\
(100-232)\end{array}$ & $\begin{array}{l}198 \\
(124-268)\end{array}$ & $2 \cdot 17$ & 13 & $\mathrm{p}<0.05$ \\
\hline
\end{tabular}

indices of capillary vaso-occlusion were used. The foveal avascular zone (FAZ) was measured as the mean of vertical and horizontal diameters and expressed as an average for both eyes. Perimacular avascular zones (PAZ) were assessed in an arbitrary circle of $2.4 \mathrm{~mm}$ diameter centred on the macula and graded from 0 (no detectable capillary drop-out) to 3 (moderate capillary drop-out). Perimacular capillary abnormalities (perimacular counts) were assessed within the same $2 \cdot 4 \mathrm{~mm}$ circle by counting the number of microaneurysms, dilated capillaries, dark and elongated arteriolar segments, and hairpin vascular loops. The total count of abnormalities in each pair of eyes was then calculated.

All angiogram assessments were made by one ophthalmologist (R.J.M.) without the knowledge of the alpha thalassaemia status of the patients.

\section{Results}

Of the 28 patients in the study complete data were not available for 3 . In one 44-year-old female patient with SS $\alpha \alpha / \alpha \alpha$ bilateral macular drusen did not allow estimates of the FAZ and PAZ although perimacular counts were available. In a 30-year-old male with SS $\alpha-/ \alpha$ - angiography of the left eye was of insufficient quality, and in a 14-year-old male with SS $\alpha \alpha / \alpha \alpha$ a left traumatic cataract precluded assessment in this eye, and angiography of the right eye was inadequate for assessment of PAZ. In the remaining 25 patients assessments of FAZ were identical in both eyes in 23, and scores for PAZ were identical in both eyes in all patients. Perimacular capillary abnormalities showed variation between the 2 eyes but the within-patient variation was considerably less than the betweenpatient variation. In view of the generally close agreement between pairs of eyes, where macular observations were unavailable in one eye the other eye was used as the best estimate of the missing observation.

The mean and range of the FAZ, PAZ, and counts of perimacular abnormalities in the 2 genotypes (SS $\alpha-/ \alpha$ - and SS $\alpha \alpha / \alpha \alpha)$ are summarised in Table 1 . Counts of the perimacular abnormalities were significantly lower in SS $\alpha-/ \alpha-$, but the size of the foveal, and grading of the perimacular, avascular zones did not differ in the 2 genotypes. None of the interrelationships between macular indices were significant.

\section{Discussion}

The syndrome resulting from the interaction of SS disease with homozygous alpha thalassaemia 2 (SS $\alpha-/ \alpha-)$ forms a valuable model for understanding the pathophysiology of SS disease. It is uncommon, accounting for only $4 \%$ of patients with SS disease attending the Jamaican clinic. For this reason and because of the defined period available for the study, it was not possible to obtain larger numbers of patients.

This subdivision of SS disease is characterised by a low mean cell volume (MCV) and a low mean corpuscular haemoglobin concentration (MCHC), both of which would be expected to inhibit intravascular sickling. The lack of a clearly protective effect against several complications with a presumed vaso-occlusive origin ${ }^{2}$ was therefore surprising and raised the possibility that the increased viscosity associated with the higher haemoglobin levels in SS $\alpha-/ \alpha$ - offset the advantages gained by inhibition of in-vivo sickling.

Previous studies of the macular vasculature in SS disease have indicated capillary drop-out with enlargement of foveal and perifoveal zones to be common, ${ }^{34}$ and an inverse relationship was noted between the size of the foveal avascular zone and the counts of perimacular capillary abnormalities. ${ }^{3}$ It was postulated that the vascular abnormalities represented an early change which disappeared with progression to total ischaemia and eventual enlargement of the foveal and perifoveal avascular zones. Counts of perimacular abnormalities therefore act as a sensitive indicator of intravascular sickling until obliteration of the vascular bed occurs. Although 
there was no evidence from the present study to indicate that capillary drop-out at the posterior pole was less frequent in SS disease with alpha thalassaemia, the lower counts of perimacular vascular abnormalities were compatible with a lesser tendency to sickling in this genotype.

\section{References}

1 Embury SH, Dozy AM, Miller J, et al. Concurrent sickle-cell anemia and $\alpha$-thalassemia: effect on severity of anemia. $N$ Engl J Med 1982; 306: 270-4.

2 Higgs DR, Aldridge BE, Lamb J, et al. The interaction of alpha thalassemia and homozygous sickle-cell disease. $N$ Engl $J$ Med 1982; 306: 1441-6.

3 Marsh RJ, Ford SM, Rabb MF, Hayes RJ, Serjeant GR. Macular vasculature, visual acuity, and irreversibly sickled cells in homozygous sickle cell disease. BrJ Ophthalmol 1982; 66: 155-60.

4 Asdourian GK, Nagpal KC, Busse B, et al. Macular and perimacular vascular remodelling in sickling haemoglobinopathies. $\mathrm{Br}$ J Ophthalmol 1976; 60: 431-53. 